\title{
The Use of Artificial Intelligence on Segmental Volumes, Constructed from MRI and CT Images, in the Diagnosis and Staging of Cervical Cancers and Thyroid Cancers: A Study Protocol for a Randomized Controlled Trial
}

\author{
Tudor Florin Ursuleanu ${ }^{1,2,3}$, Andreea Roxana Luca ${ }^{1,4}$, Liliana Gheorghe ${ }^{1,5}$, Roxana Grigorovici ${ }^{1}$, \\ Stefan Iancu ${ }^{1}$, Maria Hlusneac ${ }^{1}$, Cristina Preda ${ }^{1,6}$, Alexandru Grigorovici ${ }^{1,2}$ \\ ${ }^{1}$ Faculty of General Medicine, "Grigore T. Popa" University of Medicine and Pharmacy, Iasi, Romania; ${ }^{2 " S f}$. \\ Spiridon" Hospital, Department of Surgery VI, Iasi, Romania; ${ }^{3}$ Regional Institute of Oncology, Department of \\ Surgery I, Iasi, Romania; ${ }^{4}$ Integrated Ambulatory of Hospital "Sf. Spiridon", Department of Obstetrics and \\ Gynecology, Iasi, Romania; "Sf. Spiridon" Hospital, Department of Radiology, Iasi, Romania; “"Sf. Spiridon” \\ Hospital, Department of Endocrinology, Iasi, Romania
}

Correspondence to: Tudor Florin Ursuleanu, tudorursuleanu@yahoo.com; Andreea Roxana Luca, lucaandreearoxana@yahoo.com

Keywords: Artificial Intelligence, Cervical Cancer, Thyroid Cancer, MRI Images, CT Images

Received: May 17, $2021 \quad$ Accepted: June 25, $2021 \quad$ Published: June 28, 2021

Copyright $\odot 2021$ by author(s) and Scientific Research Publishing Inc.

This work is licensed under the Creative Commons Attribution International License (CC BY 4.0).

http://creativecommons.org/licenses/by/4.0/

\section{(c) (1) Open Access}

\section{ABSTRACT}

Rationale and Objectives: Accurately establishing the diagnosis and staging of cervical and thyroid cancers is essential in medical practice in determining tumor extension and dissemination and involves the most accurate and effective therapeutic approach. For accurate diagnosis and staging of cervical and thyroid cancers, we aim to create a diagnostic method, optimized by the algorithms of artificial intelligence and validated by achieving accurate and favorable results by conducting a clinical trial, during which we will use the diagnostic method optimized by artificial intelligence (AI) algorithms, to avoid errors, to increase the understanding on interpretation computer tomography (CT) scan, magnetic resonance imaging (MRI) of the doctor and improve therapeutic planning. Materials and Methods: The optimization of the computer assisted diagnosis (CAD) method will consist in the development and formation of artificial intelligence models, using algorithms and tools used in segmental volumetric constructions to generate $3 \mathrm{D}$ images from MRI/CT. We propose a comparative study of current developments in "DICOM" image processing by volume rendering technique, the use of the transfer function for opacity and color, shades of gray from "DICOM" images projected in a three-dimensional space. We also use artificial intelligence (AI), through the technique of Generative Adversarial Networks (GAN), which has proven 
to be effective in representing complex data distributions, as we do in this study. Validation of the diagnostic method, optimized by algorithm of artificial intelligence will consist of achieving accurate results on diagnosis and staging of cervical and thyroid cancers by conducting a randomized, controlled clinical trial, for a period of 17 months. Results: We will validate the CAD method through a clinical study and, secondly, we use various network topologies specified above, which have produced promising results in the tasks of image model recognition and by using this mixture. By using this method in medical practice, we aim to avoid errors, provide precision in diagnosing, staging and establishing the therapeutic plan in cancers of the cervix and thyroid using AI. Conclusion: The use of the CAD method can increase the quality of life by avoiding intra and postoperative complications in surgery, intraoperative orientation and the precise determination of radiation doses and irradiation zone in radiotherapy.

\section{INTRODUCTION}

Magnetic resonance imaging (MRI) and computer tomography (CT) scan play an important role in the evaluation of cervical cancer, from detection to the evaluation of recurrent disease. In medical practice, the Bethesda system [1] is used to classify thyroid cancer and the FIGO (International Federation of Gynecology and Obstetrics) system to classify cervical cancer [2]. The FIGO, Gynecological Oncology Committee recently revised its guidelines for staging cervical cancer, allowing imaging-based staging and pathological findings when available. Revised FIGO staging (2018) [2] thus allows both the selection and evaluation of therapy, the estimation of prognosis and the calculation of the final results [3].

MRI and CT can accurately evaluate prognostic indicators, for example in the cervix, tumor size, parametric invasion, pelvic lateral wall, and lymph node invasion. Despite these important roles of MRI and $\mathrm{CT}$, radiologists continue to face challenges due to the technical and interpretive pitfalls of MRI in all phases of cervical cancer and thyroid cancer assessment [4]. Also, accurate diagnosis and staging of thyroid cancer (MRI and CT images), induces the establishment of an effective therapeutic protocol. Precise measurement of thyroid volume is also necessary for the dosimetry of radioactive iodine therapies and the evaluation of the therapeutic effect. In surgical management, thyroid size has become a requirement in selecting patients for minimally invasive surgery [4].

The novelty of our research consists of two elements: firstly, by the fact that we validate the computer assisted diagnostic (CAD) method through a clinical study and, secondly, we use various network topologies specified above, which have produced promising results in the tasks of image model recognition and by using this mixture, we will specialize this network mix by requalifying the last layers with field specific images-MRI and CT scan. We aim to create a CAD method optimized by the algorithms of artificial intelligence and validated by accurate results on diagnosis and staging of cervical precancers and by conducting a randomized, controlled clinical trial. We will validate the CAD method by the results of the clinical trial, randomized, controlled, carried out over the 17-month period.

Automatic diagnosis of images collected using a three-dimensional MRI and CT reconstruction algorithm can avoid errors, provide precision in viewing information, and create the prerequisites for a superior relationship between data and the establishment of therapeutic protocol, with defined application in surgery, gynecology, oncology and radiotherapy.

\section{MATERIALS AND METHOD}

We aim to create a computer assisted diagnosis (CAD) method optimized by the algorithms of artificial intelligence and validated by accurate results on diagnosis and staging of cervical and thyroid cancers by conducting a randomized, controlled clinical trial.

The optimization of the CAD method will consist in development and pre-training of artificial intel- 
ligence algorithms for optimization the diagnosis and staging of cervical and thyroid cancer through imaging investigations MRI, CT.

The main analysis will focus on the automatic diagnosis of the collected images using a three-dimensional MRI and CT reconstruction algorithm. We propose for the beginning a comparative study of the current developments in image processing in "DICOM" format through the technique of volume rendering $[5,6]$.

"DICOM" [7] is currently the most common standard for the transmission of medical images and other patient information. "DICOM" accepts a wide range of medical images, including CT and MRI as well as medical images in the fields of radiology, cardiology, pathology, dentistry and other fields. The "DICOM" standard provides for storage in a directory that contains several files in "DICOM" format and organizes them by patient, study and series.

We will synthesize the theory underlying the volume rendering technique, listing some algorithms based on the discretization of the physical process that models the passage of light through permeable material $[5,6]$. By using a so-called transfer function for opacity and color, the shades of gray images are projected into a three-dimensional space used "DICOM" [8].

We will follow a set of quantitative and qualitative parameters of software applications and libraries on the market such as: minimum hardware requirements, licensing mode (open source vs commercial), portability between various operating systems, ease of installation and use, quality of volumetric interpretations, speed of interpretation, maintenance and support provided by the development team, extensibility through existing connectors or custom code, quality of documentation and availability of training in the use of software products.

Ultimately, we will study a new approach that recognizes the challenges of configuring and using transfer functions to adjust the optical properties of 3D interpretation. The surgeon's goal is to understand the distinctive features of volumetric interpretation, which is not always possible by simply limiting ourselves to transfer functions, requiring a detailed inspection of these three-dimensional spaces. Artificial intelligence (AI), through the technique of Generative Adversarial Networks (GAN) has proven to be effective in representing complex data distributions [9], as we do in the present study.

Validation of the method will consist in achieving accurate and favorable results by conducting a randomized, controlled, clinical trial, for a period of 17 months, during which we will use the diagnostic method optimized by artificial intelligence (AI) algorithms in the diagnosis and staging of cervical and thyroid cancers.

Patients with cervical and thyroid cancer will be included in the clinical trial. Patients will be informed and recruited through random presentation at the specialized medical centers nominated in the clinical trial and through a web platform created for this purpose. We propose the inclusion of 60 patients in the clinical trial. We will create a control lot and a study lot.

The two-branch control group (control group with cervix cancer-G1c and group control with thyroid cancer-G1t) will consist of patients who will be diagnosed and staging (MRI and CT scan) without using the CAD method by artificial intelligence algorithms.

The two-branch study group (study group with cervix cancer-G2c and study group with thyroid cancer-G2t) will consist of patients who will be diagnosed using the CAD method, but without the involvement of the investigator.

The key control of diagnostic and staging of patients in the control group and the study group will consist of the anatomopathological result of the patient (surgical excision piece). We will differentiate the study and control groups respectively: cervix, G1c and G2c and for thyroid G1t and G2t respectively.

Inclusion criteria: in the control and study groups G1c and G2c for the cervix, patients with precancerous staging; in the control and study groups (G1t and G2t) for thyroid, patients with diagnosis - staging thyroid cancer stages 0 -IV.

Exclusion criteria: in the G1c and G2c batches we exclude the patients virgo intacta, patient's personal history (A.P.) total hysterectomy, A.P. trachelectomy, pregnancy, comorbidities incompatible with the clinical trial; in the G1t and G2t groups we exclude benign pathologies, thyroidectomized patients, comorbidities incompatible with the clinical trial. 
We will use the Bethesda system [1] for the classification of thyroid cancer and FIGO system [2] for the classification cervical cancer.

MRI and CT images represent by their interpretation, diagnosis and staging of cervical and thyroid cancers.

We will use the binary model $0 / 1$, to note with " 0 ", lack of correspondence and " 1 " respectively correspondence, between diagnosis and staging performed by the investigator compared to the diagnosis and staging performed with the support of AI, for cervical cancer and thyroid cancer.

We will also use the same binary model to assess diagnostic correspondence and staging of the investigator and the CAD method with the anatomopathological diagnosis (surgical excision piece) and will be the key to diagnostic control and staging for the practitioner and for the CAD method.

Diagnostic and staging accuracy of the CAD method will result from its diagnostic correspondence with the investigator's and the anatomopathological diagnosis. Patients will be requested to sign informed standard consent to participate in the clinical trial and will be able to leave the clinical trial at any time without providing an explanation and without repercussions on treatment.

\section{RESULTS}

We will validate the computer assisted diagnostic method through a clinical study and, secondly, we use various network topologies specified above, which have produced promising results in the tasks of image model recognition and by using this mixture we will specialize this network mix by requalifying the last layers with field. By using this CAD method in medical practice, we aim to avoid errors, provide precision in diagnosing, staging and establishing the therapeutic plan in cancers of the cervix and thyroid using AI.

\section{DISCUSSION}

Most computer assisted diagnostic methods are not validated by clinical trials, and researchers have recently turned their attention to this issue. Also, the mix of deep learning architectures we use for the CAD method is a novelty. Compared to previous studies, the novelty in our research includes: creating the CAD method using network topologies mix and validate the CAD method through a clinical study. We will undertake measures to ensure patient's safety, for an effective therapeutic result and for patient's compliance, in concordance with ethical protocol, carried out through specific activities within the framework of scientific research and are main objectives for avoiding the risks of failure and for the successful implementation and completion of the research project.

\section{CONCLUSION}

This CAD method, optimized by artificial intelligence algorithms and validated by the clinical study, considered the "second opinion" of diagnosis. CAD method improves the quality standard in diagnosis, staging and establishment of therapeutic conduct in cervical and thyroid cancers, with defined application in surgery, gynecology, oncology and radiotherapy. The use of the CAD method can increase the quality of life by avoiding intra and postoperative complications in surgery, intraoperative orientation and the precise determination of radiation doses and irradiation zone in radiotherapy.

\section{FUNDING}

Scientific research funded by the University of Medicine and Pharmacy "Gr. T. Popa" of Iasi, based on contract number 4714 .

\section{CONFLICTS OF INTEREST}

None declared. 


\section{REFERENCES}

1. Upadhyaya, P., Dhakal, S., Adhikari, P., Adhikari, B., Khadka, D. and Niraula, S.R. (2019) Histopathological Review of Diagnostic Categories of the Bethesda System for Reporting Thyroid Cytopathology-An Institutional Experience of 5 Years. Journal of Cytology, 36, 48-52. https://doi.org/10.4103/JOC.JOC 6418

2. Liu, X., Wang, J., Hu, K., Zhang, F., Meng, Q., Wang, W., Wang, D., Zhou, Z. and Ren, K. (2020) Validation of the 2018 FIGO Staging System of Cervical Cancer for Stage III Patients with a Cohort from China. Cancer Management and Research, 12, 1405-1410. https://doi.org/10.2147/CMAR.S239624

3. Horn, L.C., Brambs, C.E., Opitz, S., Ulrich, U.A. and Höhn, A.K. (2019) The 2019 FIGO Classification for Cervical Carcinoma-What's New? Der Pathologe, 40, 629-635. https://doi.org/10.1007/s00292-019-00675-w

4. Otero-García, M.M., Mesa-Álvarez, A., Nikolic, O., Blanco-Lobato, P., Basta-Nikolic, M., de Llano-Ortega, R.M., Paredes-Velázquez, L., Nikolic, N. and Szewczyk-Bieda, M. (2019) Role of MRI in Staging and Follow-Up of Endometrial and Cervical Cancer: Pitfalls and Mimickers. Insights Imaging, 10, 19. https://doi.org/10.1186/s13244-019-0696-8

5. Eghtedari, M., Chong, A., Rakow-Penner, R. and Ojeda-Fournier, H. (2021) Current Status and Future of BI-RADS in Multimodality Imaging, from the AJR Special Series on Radiology Reporting and Data Systems. American Journal of Roentgenology, 216, 860-873. https://doi.org/10.2214/AJR.20.24894

6. Ketkar, N. and Santana, E. (2017) Deep Learning with Python. Vol. 1, Springer, Berkeley, CA. https://doi.org/10.1007/978-1-4842-2766-4__1

7. DICOM-Logo Search. https://www.dicomstandard.org

8. Berger, M., Li, J. and Levine, J.A. (2018) A Generative Model for Volume Rendering. IEEE Transactions on Visualization and Computer Graphics, 25, 1636-1650. https://doi.org/10.1109/TVCG.2018.2816059

9. Driss, S.B., Soua, M., Kachouri, R. and Akil, M. (2017) A Comparison Study between MLP and Convolutional Neural Network Models for Character Recognition. SPIE Conference on Real-Time Image and Video Processing, Anaheim, CA, April 2017. https://doi.org/10.1117/12.2262589 